\title{
Optimización de las propiedades mecánicas de las aleaciones AISi7Mg moldeadas en arena
}

\author{
A. Loizaga*, E. de la Fuente*, A. Niklas*, J. Barrenengoa** y A.I. Fernández-Calvo*
}

\begin{abstract}
Resumen Se han estudiado las características mecánicas de la aleación AlSi7Mg, con objeto de elegir la composición y el tratamiento térmico idóneos para el moldeo de piezas en arena. Las propiedades mecánicas han sido evaluadas variando el contenido de magnesio, cubriendo tanto la aleación A356 como la A357. La incidencia del tratamiento térmico se ha estudiado en la aleación A356 variando la temperatura y el tiempo de precipitación, manteniendo constante el tratamiento de disolución. Se han definido distintos índices de calidad que combinan resistencia mecánica, límite elástico y alargamiento, permitiendo seleccionar un tratamiento térmico y una composición química adecuada para cada aplicación.
\end{abstract}

\section{Mechanical properties optimization of alloys AISi7Mg casting in sand moulds}

\begin{abstract}
In the present work the mechanical properties of the alloy AlSi7Mg were studied in order to choose a suitable composition and heat treatment for sand castings. The mechanical properties have been evaluated varying the content of $\mathrm{Mg}$, covering the alloys A356 and A357. The effect of heat treatment has been studied in alloy A356 varying the emperature and time of the precipitation heat treatment, while the dissolution treatment was kept constant. Several quality indexes have been defined which combine tensile strength, yield strength, tensile strength and elongation in order to select an adequate chemical composition and heat treatment for each application.
\end{abstract}

Keywords

A356 and A357 aluminium alloys; Mechanical properties; Effect of Mg content; Effect of heat treatment T6.

\section{INTRODUCCIÓN}

Las aleaciones $\mathrm{Al}-\mathrm{Si}-\mathrm{Mg}$ se caracterizan por su buena colabilidad y la posibilidad de obtener buenas propiedades mecánicas, en piezas fabricadas en fundición por gravedad.

En la práctica habitual para obtener buenas propiedades mecánicas se aplican los siguientes tratamientos al metal líquido: afino de grano, mediante la adición de aleaciones madre, base Ti-B, modificación del silicio eutéctico, mediante adición de estroncio y/o sodio ${ }^{[1-9]}$ y desgasificado, para reducir el nivel de hidrógeno en el metal líquido.

Principalmente, es el tratamiento térmico el que confiere las propiedades mecánicas finales, pudiéndose elegir dentro de un amplio rango de valores ${ }^{[10-14]}$. Existe amplia documentación sobre las correlaciones de los parámetros del tratamiento térmico T6 y las propie- dades mecánicas ${ }^{[10-15]}$, mayoritariamente en moldes metálicos.

Conviene señalar que las propiedades mecánicas son inferiores, en piezas fabricadas en moldes de arena respecto a las fabricadas en moldes metálicos, como consecuencia de la menor velocidad de solidificación. Las mejoras en los tratamientos del metal, en los últimos años, permiten alcanzar buenos resultados tanto en carga de rotura como en alargamiento, de forma que se pueden utilizar piezas moldeadas en arena para aplicaciones de alta responsabilidad mecánica.

Teniendo en cuenta que el tratamiento térmico T6 tiene por objeto la disolución y posterior precipitación en solución sólida de las partículas de $\mathrm{Mg}_{2} \mathrm{Si}$, su efectividad depende, fuertemente, de la concentración del magnesio aleado. En el presente trabajo se ha estudiado:

\footnotetext{
*AZTERLAN, Ingeniería y Procesos de Fundición Dpt., Aliendalde Auzunea, 6, E-48200, Durango. e-mail: afernandez@azterlan.es.

** TQC Technologies, Aliendalde Auzunea, 10, E-48200, Durango. e-mail: tqctechnologies@gmail.com.
} 
- Efecto del tratamiento térmico: Se han analizado diferentes tiempos y temperaturas de precipitación para un contenido de magnesio, de $0,29 \%$.

- Efecto del contenido del magnesio en la aleación AlSi7Mg, bajo idéntico tratamiento térmico, T6, cubriéndose el rango de composición de las aleaciones A356 y A357.

La optimización de las propiedades mecánicas se realiza en base a la comparativa con diferentes índices de calidad: $R_{\mathrm{m}} \times \mathrm{A}, R_{\mathrm{p} 0,2} / R_{\mathrm{m}}$ y el índice de calidad $Q$, siendo $Q=R_{m}+K \cdot \log A$, que relaciona la carga de rotura $\left(R_{\mathrm{m}}\right)$, con el alargamiento $(A)$ mediante el coeficiente $K^{[16}$ y 17$]$. El parámetro $K$ es un coeficiente empírico que se ha seleccionado de manera que el parámetro $Q$ no dependa del tratamiento térmico. Para la aleación AlSi7Mg se ha determinado el valor de $150 \mathrm{MPa}$. La dependencia de $Q$ con el alargamiento se muestra en la figura 1 . El índice $R_{\mathrm{m}} \times$ A está relacionado con la energía a rotura del material, mientras que $Q$ es una variante del anterior, de uso habitual en la industria. El índice $R_{\mathrm{p} 0,2} / R_{\mathrm{m}}$ es independiente de los parámetros anteriores, no teniendo en cuenta el alargamiento y sirve como indicador de la optimización del material en relación a la resistencia mecánica.

\section{MÉTODO EXPERIMENTAL}

Para el presente trabajo se ha seleccionado la aleación de aluminio AlSi7Mg. Los ensayos se han agrupado en dos series:

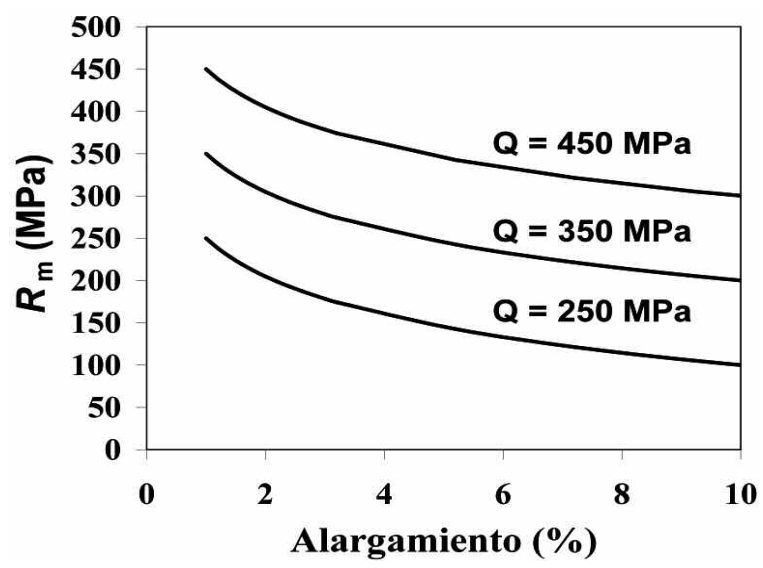

Figura 1. Dependencia del factor de calidad, $Q$, con el alargamiento (coeficiente $\mathrm{K}=150 \mathrm{MPa}$ ).

Figure 1. Dependence of the $Q$ quality factor, with elongation (coefficient $K=150 \mathrm{MPa}$ ).

- Serie I: Se ha seleccionado la aleación que aparece en la tabla I (aleación I), con un contenido en magnesio de 0,29\%, para el estudio de la influencia del tiempo y temperatura de precipitación en el tratamiento térmico, T6.

- Serie II: Se analiza el efecto del contenido en magnesio, entre 0,28 y $0,67 \%$, en las propiedades mecánicas, bajo un mismo tratamiento térmico, T6: solubilización a $540{ }^{\circ} \mathrm{C}$ durante $8 \mathrm{~h}$, seguido de temple en agua a $20^{\circ} \mathrm{C}$ y tratamiento de precipitación a $155^{\circ} \mathrm{C}$ durante 5,5 h. El rango de composición química analizado cubre las aleaciones A356 y A357 (serie II, Tabla I).

Tabla I. Composición química de las aleaciones AISi7Mg analizadas

Table I. Chemical composition of the analyzed AISi7Mg alloys

\begin{tabular}{ccccccccc}
\hline \multirow{2}{*}{ Series } & \multicolumn{7}{c}{ Composición química (\% en peso) } \\
\cline { 2 - 9 } & $\mathbf{S i}$ & Fe & \multicolumn{1}{c}{ Cu } & Mn & Mg & Zn & Ti & Sr \\
\hline \multirow{2}{*}{$\mathbf{I}$} & 6,50 & 0,15 & $<0,01$ & 0,01 & 0,29 & $<0,01$ & 0,15 & 0,010 \\
& 6,75 & 0,10 & $<0,01$ & 0,06 & 0,28 & $<0,01$ & 0,19 & 0,005 \\
& 7,06 & 0,10 & $<0,01$ & 0,01 & 0,29 & $<0,01$ & 0,17 & 0,007 \\
& 6,82 & 0,09 & $<0,01$ & $<0,01$ & 0,31 & $<0,01$ & 0,15 & 0,008 \\
II & 6,66 & 0,09 & $<0,01$ & 0,04 & 0,33 & $<0,01$ & 0,13 & 0,011 \\
& 7,35 & 0,18 & $<0,03$ & $<0,01$ & 0,46 & $<0,01$ & 0,16 & 0,010 \\
& 6,67 & 0,11 & $<0,01$ & 0,04 & 0,54 & $<0,01$ & 0,18 & 0,010 \\
& 6,85 & 0,12 & $<0,01$ & 0,05 & 0,55 & $<0,01$ & 0,17 & 0,009 \\
& 6,73 & 0,10 & $<0,01$ & 0,01 & 0,60 & $<0,01$ & 0,15 & 0,012 \\
& 6,80 & 0,11 & $<0,01$ & 0,05 & 0,67 & $<0,01$ & 0,17 & 0,012 \\
\hline
\end{tabular}


Con objeto de obtener buenas propiedades mecánicas, todas las aleaciones han sido sometidas a tratamientos de afino de grano mediante la adición de aleación madre AlTi5B1 (200 g, por cada $100 \mathrm{~kg}$ de metal) y tratamiento de modificado del silicio eutéctico mediante la adicción de estroncio $(0,005$. $0,012 \%)$. El buen grado de afino y de modificado ha sido comprobado, en todas las coladas, haciendo uso

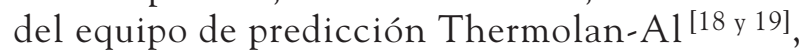
basado en la predicción de las características estructurales en la taza de caracterización térmica, mediante el registro de la curva de solidificación.

En todas las coladas se ha reducido el nivel de hidrógeno del metal líquido, mediante borboteo de nitrógeno gaseoso, hasta alcanzarse el valor de densidad mínimo, de $2,65 \mathrm{~g} / \mathrm{cm}^{3}$, en probetas solidificadas en vacío. Una vez tratado el metal, como se ha indicado previamente, se cuelan varias probetas de tracción, en moldes de arena aglomerada químicamente, a temperaturas entre 710 y $750{ }^{\circ} \mathrm{C}$.

La aleación I se ha fabricado en una empresa de fundición, con metal procedente de su línea de producción, mientras que la serie II se ha realizado en la planta de fundición experimental TQC Technologies.

La microestructura, esto es, tamaño de grano, grado de modificado, tamaño y fases presentes y porosidad se ha evaluado sobre la zona de amarre de las probetas de tracción en bruto de colada. El tratamiento térmico T6 se ha aplicado en un horno de laboratorio, con control de temperatura de $\pm 2{ }^{\circ} \mathrm{C}$. Posteriormente, se han mecanizado las probetas de tracción, con $10 \mathrm{~mm}$ de diámetro y se han realizado los ensayos según procedimiento establecido en la norma UNE-EN_1706.

\section{RESULTADOS Y DISCUSIÓN}

En todas las coladas se han obtenido buenas características estructurales, tal y como predecía el sistema Thermolan-Al mediante el registro de la curva de solidificación. Todas las probetas muestran tamaños de grano inferiores a $0,55 \mathrm{~mm}$, grados de modificado entre 3 y 4 ; y porosidad inferior a 0,35\%. Las partículas de fase $\mathrm{b}-\mathrm{Al}_{5} \mathrm{FeSi}$ presentan tamaños inferiores a $50 \mu \mathrm{m}$. En la figura 2 se muestra, a modo de ejemplo, una de las características microestructurales más significativas de la aleación I.

\subsection{Incidencia del tratamiento térmico}

En este apartado se estudia la influencia del tratamiento térmico sobre las propiedades mecánicas de la aleación $\operatorname{AlSi} 7 \mathrm{Mg}(0,29 \%$ magnesio), mediante
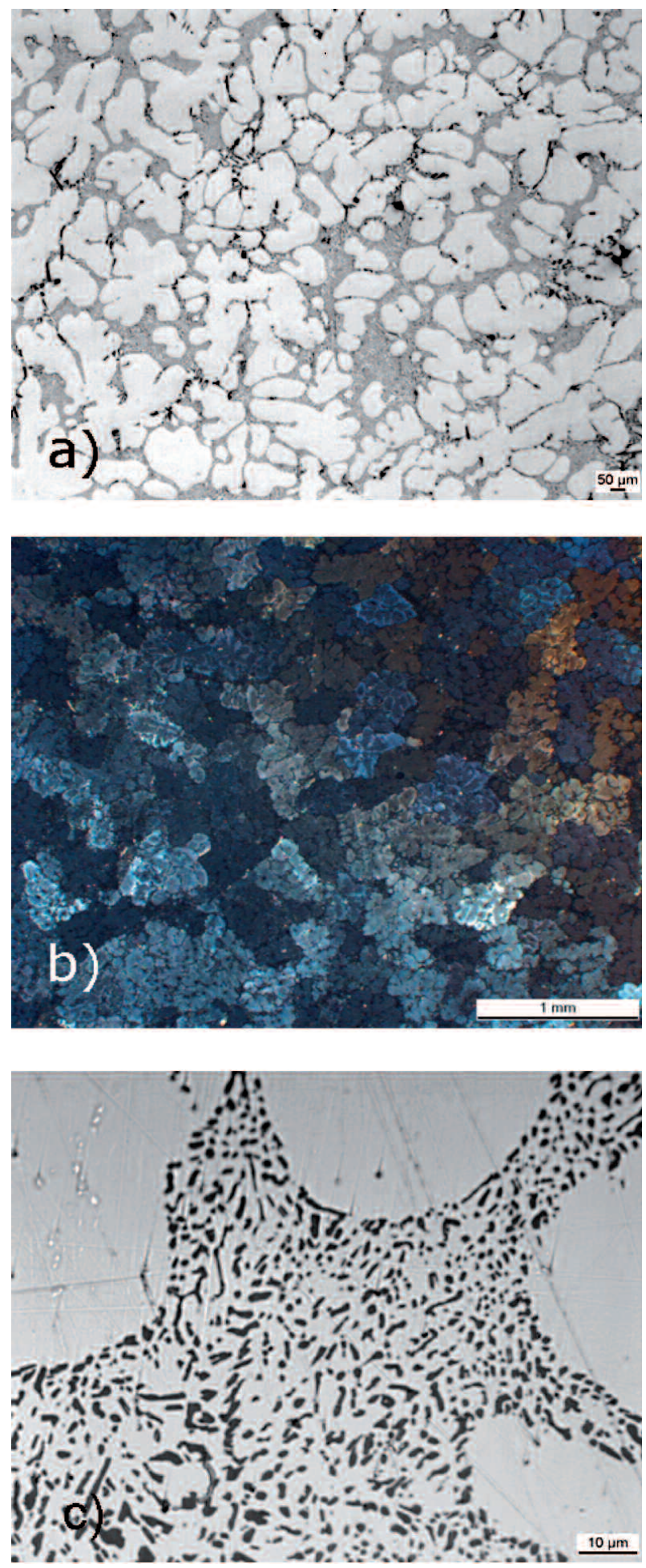

Figura 2. Características estructurales típicas de la aleación AISi7Mg en bruto de colada: (a) Aspecto general de las dendritas de aluminio; (b) Tamaño de grano; (c) grado de modificado para la aleación I.

Figure 2. Typical structural characteristics of AlSi7Mg alloys in as-cast state: (a) General aspect of aluminium dendrites; (b) Grain size; (c) Modification grade for the alloy $I$. 
la realización de una serie de ensayos en los que se varió la temperatura y el tiempo de precipitación. El tratamiento térmico, además de disolver y, posteriormente, precipitar la fase $\mathrm{Mg}_{2} \mathrm{Si}$, globuliza el silicio eutéctico y redondea las fases , $-\mathrm{Al}_{5} \mathrm{FeSi}$, mejorando, por todos estos motivos, las propiedades mecánicas.

En la tabla II y la figura 3 se muestran los resultados de las propiedades mecánicas y los indicadores de calidad para los tratamientos realizados a 155,160 y 170 ${ }^{\circ} \mathrm{C}$, durante diferentes tiempos de precipitación, entre 3,5 y 6,5 h. Se observan los siguientes resultados:

- Para un mismo tiempo de tratamiento, a menor temperatura de precipitación, menor son límite elástico, carga máxima y dureza; mientras que la ductilidad aumenta.

- A igualdad de temperatura de tratamiento, conforme se incrementa el tiempo de permanencia entre 3,5 y 6,5 h, límite elástico, carga máxima y dureza aumentan, mientras que el alargamiento decrece.

El ratio $R_{\mathrm{p} 0,2} / R_{\mathrm{m}}$ aumenta conforme aumenta la temperatura y el tiempo de permanencia, lo que significa un mayor aprovechamiento del material (Tabla II). En la figura 4 se muestran los valores de carga máxima frente al alargamiento y las rectas correspondientes a los valores del índice de calidad $\mathrm{Q}=370,380$ y $390 \mathrm{MPa}$. Todas las parejas de valores $\left(R_{\mathrm{m}}, \mathrm{A}\right)$ del presente trabajo están comprendidas

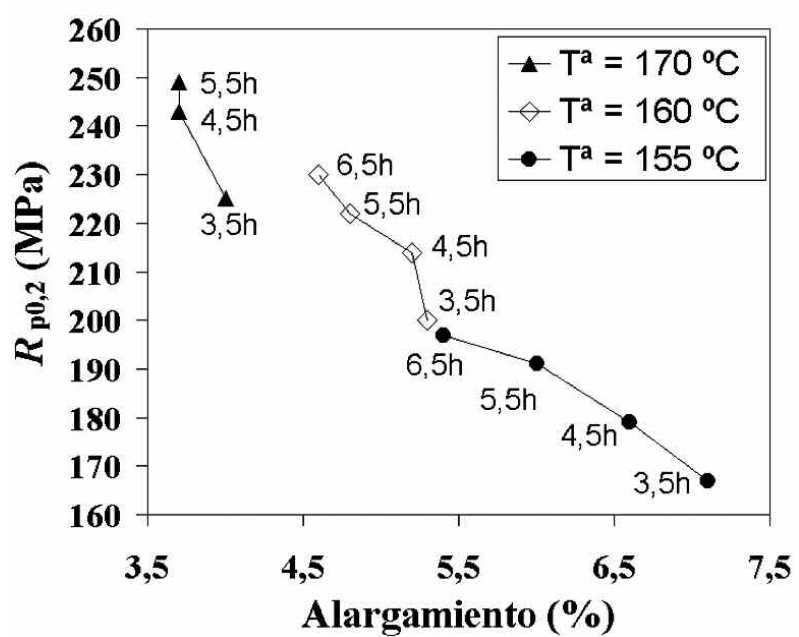

Figura 3. Límite elástico y carga a rotura frente al alargamiento, para diferentes temperaturas y tiempos de precipitación de la aleación I.

Figure 3. Yield strength and maximum load vs. elongation for different temperatures and precipitation times of the alloy $I$.

en este rango de índices de calidad. Los valores máximos de $Q$ responden a la temperatura de precipitación de $160^{\circ} \mathrm{C}$ (excepto $3,5 \mathrm{~h}$ ), siendo muy próximos, también, los valores obtenidos a $155^{\circ} \mathrm{C}$. Teniendo en cuenta el ratio $R_{\mathrm{m}} \times \mathrm{A}$, los mejores valores responden a la temperatura de $155^{\circ} \mathrm{C}$. De

Tabla II. Propiedades mecánicas e índices de calidad para la aleación I a diferentes temperaturas y tiempos de precipitación

Table II. Mechanical properties and quality factors for the alloy I at different temperatures and precipitation times

\begin{tabular}{|c|c|c|c|c|c|c|c|c|}
\hline \multicolumn{2}{|c|}{ Maduración } & \multicolumn{4}{|c|}{ Propiedades Mecánicas } & \multicolumn{3}{|c|}{ Indices de Calidad } \\
\hline $\begin{array}{c}\mathrm{T}^{\mathrm{a}} \\
\left({ }^{\circ} \mathrm{C}\right)\end{array}$ & $\begin{array}{c}\text { Tiempo } \\
\text { (h) }\end{array}$ & $\begin{array}{c}R_{\mathrm{m}} \\
(\mathrm{MPa})\end{array}$ & $\begin{array}{c}R_{\mathrm{p} 0,2} \\
(\mathrm{MPa})\end{array}$ & $\begin{array}{c}A \\
(\%)\end{array}$ & $\begin{array}{c}\text { Dureza } \\
\text { (HB) }\end{array}$ & $\begin{array}{c}R_{\mathrm{p} 0,2} I \\
R_{\mathrm{m}}\end{array}$ & $\begin{array}{c}R_{\mathrm{m}} \times A \\
(\mathrm{MPa} \% \%)\end{array}$ & $\begin{array}{c}Q \\
(\mathrm{MPa})\end{array}$ \\
\hline 155 & $\begin{array}{l}3,5 \\
4,5 \\
5,5 \\
6,5\end{array}$ & $\begin{array}{l}254 \\
260 \\
267 \\
269\end{array}$ & $\begin{array}{l}167 \\
179 \\
191 \\
197\end{array}$ & $\begin{array}{l}7,1 \\
6,6 \\
6,0 \\
5,4\end{array}$ & $\begin{array}{l}79,5 \\
81,5 \\
84,5 \\
87,5\end{array}$ & $\begin{array}{l}0,66 \\
0,69 \\
0,72 \\
0,73\end{array}$ & $\begin{array}{l}1.806 \\
1.716 \\
1.602 \\
1.453\end{array}$ & $\begin{array}{l}382 \\
383 \\
384 \\
379\end{array}$ \\
\hline 160 & $\begin{array}{l}3,5 \\
4,5 \\
5,5 \\
6,5\end{array}$ & $\begin{array}{l}269 \\
278 \\
283 \\
289\end{array}$ & $\begin{array}{l}200 \\
214 \\
222 \\
230\end{array}$ & $\begin{array}{l}5,3 \\
5,2 \\
4,8 \\
4,6\end{array}$ & $\begin{array}{l}88,0 \\
89,0 \\
93,7 \\
91,6\end{array}$ & $\begin{array}{l}0,74 \\
0,77 \\
0,78 \\
0,80\end{array}$ & $\begin{array}{l}1.426 \\
1.446 \\
1.358 \\
1.329\end{array}$ & $\begin{array}{l}378 \\
385 \\
385 \\
388\end{array}$ \\
\hline 170 & $\begin{array}{l}3,5 \\
4,5 \\
5,5\end{array}$ & $\begin{array}{l}284 \\
294 \\
294\end{array}$ & $\begin{array}{l}225 \\
243 \\
249\end{array}$ & $\begin{array}{l}4,0 \\
3,7 \\
3,7\end{array}$ & $\begin{array}{l}94,0 \\
98,0 \\
97,0\end{array}$ & $\begin{array}{l}0,79 \\
0,83 \\
0,85\end{array}$ & $\begin{array}{l}1.136 \\
1.088 \\
1.088\end{array}$ & $\begin{array}{l}374 \\
379 \\
379\end{array}$ \\
\hline
\end{tabular}




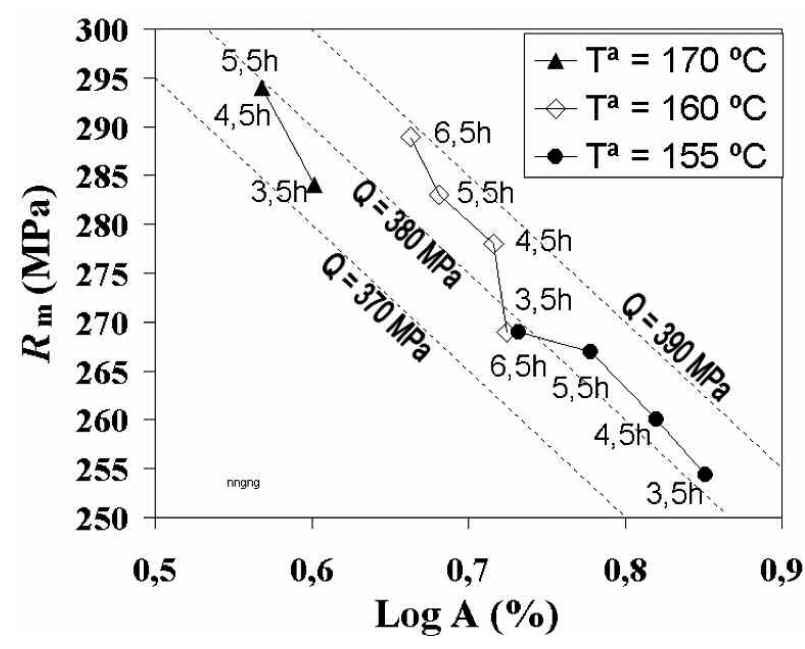

Figura 4. Carga máxima frente alargamiento para diferentes temperaturas y tiempos de precipitación de la aleación I. Se indican los índices de calidad $Q$ entre 370 y $390 \mathrm{MPa}$, calculados con $\mathrm{K}=150 \mathrm{MPa}$.

Figure 4. Maximum load vs. elongation for different temperatures and precipitation times of the alloy I. The $Q$ quality factors between 370 and $390 \mathrm{MPa}$ calculated with $\mathrm{K}=150 \mathrm{MPa}$ are indicated.

acuerdo con estos resultados, se puede considerar el tratamiento de maduración a $155^{\circ} \mathrm{C}$, durante $5,5 \mathrm{~h}$, como el tratamiento con el que se consigue un buen equilibrio entre las propiedades mecánicas y los índices de calidad, además de presentar valores de alargamientos elevados. Estos han sido los motivos de su selección para estudiar el efecto del contenido en magnesio, en el apartado siguiente.

Por otro lado, teniendo en cuenta que se observa una dependencia cuasi-lineal entre el límite elástico y el alargamiento se puede establecer el tratamiento térmico óptimo para esta aleación, en función de las necesidades de resistencia y ductilidad.

\subsection{Influencia del magnesio aleado}

Para el estudio de la influencia del contenido del magnesio, se han seleccionado las condiciones de tratamiento térmico T6: Solubilización a $540^{\circ} \mathrm{C}$ durante $8 \mathrm{~h}$, temple a $20^{\circ} \mathrm{C}$ y maduración a $155^{\circ} \mathrm{C}$ durante $5,5 \mathrm{~h}$, tal y como se ha comentado en el apartado precedente. En la figura 5 se muestran las curvas tensióndeformación, a temperatura ambiente, de la aleación AlSi7Mg para tres contenidos de magnesio diferentes.

Los valores medios de límite elástico, carga máxima y alargamientos obtenidos para las aleaciones de esta

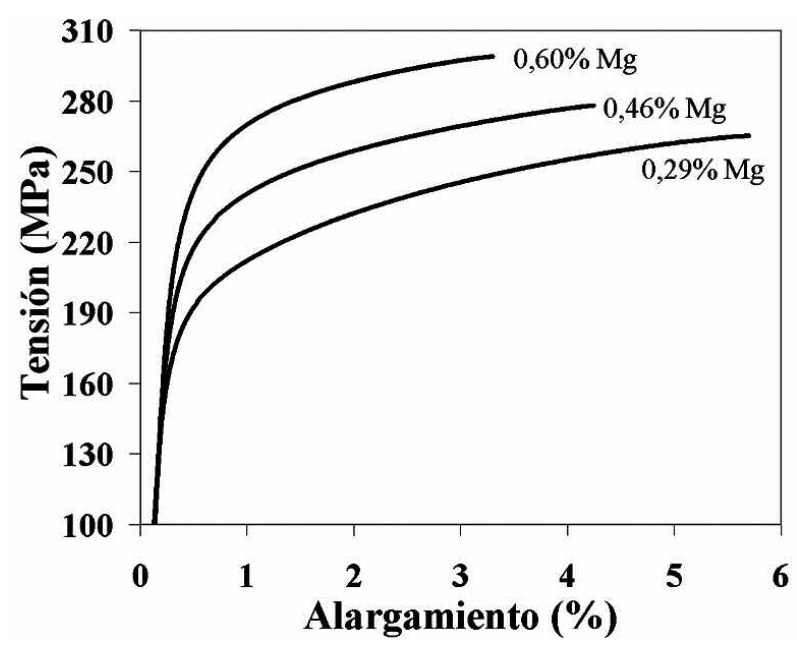

Figura 5. Curvas tensión-deformación de varias aleaciones de la serie II después de tratamiento térmico T6 $\left(540{ }^{\circ} \mathrm{C}, 8 \mathrm{~h}-155^{\circ} \mathrm{C}, 5,5 \mathrm{~h}\right)$.

Figure 5. Stress-strain curves of several alloys of the series II after the heat treatment T6 $\left(540^{\circ} \mathrm{C}, 8 \mathrm{~h}-155^{\circ} \mathrm{C}, 5,5 \mathrm{~h}\right)$.

serie II se recogen en la tabla III y se representan en la figura 6 , frente al contenido de magnesio. Se observa que, al aumentar el contenido en magnesio (aleación AlSi7Mg) se produce la disminución del alargamiento, a la vez que aumenta el límite elástico y la carga a rotura. En la figura 7 se pone de manifiesto, gráficamente, el efecto del magnesio, tanto en el límite elástico como en la carga rotura.

En la figura 6 se muestran, también, las ecuaciones de ajuste (1) y (2), de límite elástico y alargamiento, en función del contenido en magnesio para el tratamiento T6. De esta forma, en función de los requerimientos de la pieza en cuanto a límite elástico y ductilidad, se puede estimar el contenido de magnesio óptimo en función del límite elástico y alargamiento requeridos.

$$
\begin{gathered}
R_{p o, 2}(\mathrm{MPa})=-335[\% \mathrm{Mg}]^{2}+440[\mathrm{Mg}]+94 \\
A(\%)=12[\% \mathrm{Mg}]^{2}-20[\mathrm{Mg}]+10
\end{gathered}
$$

En la tabla III, se observa, claramente, cómo el aumento del contenido en magnesio, aumenta el ratio $R_{\mathrm{p} 0,2} / R_{\mathrm{m}}$, mientras los parámetros de calidad $R_{\mathrm{m}} \times \mathrm{A}$ y $Q$ descienden, influidos, sobre todo, por el descenso del alargamiento. En la figura 8, se puede observar como la dispersión de valores en el parámetro $R_{\mathrm{m}} \times \mathrm{A}$ es menor que en el parámetro de calidad Q (mayor coeficiente de determinación, $R^{2}$ ), siendo el contenido de magnesio, próximo a 0,30 \%, el que proporciona los valores máximos de ambos parámetros 
Tabla III. Propiedades mecánicas y parámetros de las aleaciones AISi7Mg de la serie II, después de tratamiento térmico T6 $\left(540{ }^{\circ} \mathrm{C}, 8 \mathrm{~h}-155{ }^{\circ} \mathrm{C}, 5,5 \mathrm{~h}\right)$

Table III. Mechanical properties and parameters of the alloys AISi7Mg of the serie II, after the heat treatment $\mathrm{T} 6\left(540{ }^{\circ} \mathrm{C}, 8 \mathrm{~h}-155^{\circ} \mathrm{C}, 5,5 \mathrm{~h}\right)$

\begin{tabular}{ccccccc}
\hline Ref. (\%Mg) & $\mathbf{R}_{\mathbf{m}}$ (MPa) & $\mathbf{R}_{\mathbf{p} 0,2}(\mathbf{M P a})$ & $\mathbf{A}(\%)$ & $\mathbf{R}_{\mathbf{p} 0,2} / \mathbf{R}_{\mathbf{m}}$ & $\mathbf{R}_{\mathbf{m}} \times \mathbf{A}(\mathbf{M P a} \%)$ & $\mathbf{Q}(\mathbf{M P a})$ \\
\hline 0,28 & 262 & 181 & 5,9 & 0,69 & 1.546 & 378 \\
0,29 & 253 & 186 & 4,4 & 0,74 & 1.113 & 350 \\
0,29 & 267 & 191 & 6,0 & 0,71 & 1.602 & 384 \\
0,31 & 284 & 217 & 4,5 & 0,76 & 1.278 & 382 \\
0,33 & 273 & 202 & 5,5 & 0,73 & 1.501 & 384 \\
0,46 & 276 & 217 & 3,7 & 0,78 & 1.021 & 361 \\
0,54 & 281 & 233 & 2,2 & 0,83 & 618 & 332 \\
0,55 & 285 & 234 & 2,5 & 0,82 & 713 & 345 \\
0,60 & 281 & 240 & 2,9 & 0,85 & 815 & 350 \\
0,67 & 282 & 237 & 1,9 & 0,84 & 536 & 324 \\
\hline
\end{tabular}

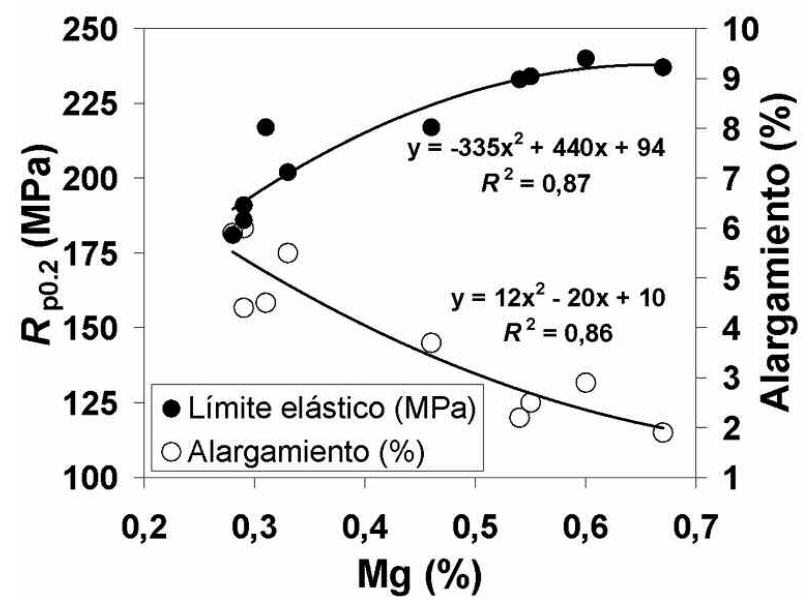

Figura 6. Variación del límite elástico y alargamiento en función del porcentaje de magnesio, después de tratamiento térmico T6, para la serie II.

Figure 6. Yield strength and elongation as a function of magnesium content, after heat treatment T6 for the series II.

aunque, para un contenido de magnesio de 0,3\%, se obtienen los valores del ratio $R_{\mathrm{p} 0,2} / R_{\mathrm{m}}$ menores (Tabla III), lo que estaría indicando un bajo aprovechamiento del material, en estas condiciones. En el presente trabajo, no se han encontrado las condiciones de aleación (porcentaje de magnesio) o tratamiento térmico que maximicen los tres índices de calidad, de forma simultanea. Sin embargo, en función del índice elegido, se puede definir tanto el contenido en magnesio como el tratamiento térmico óptimo.

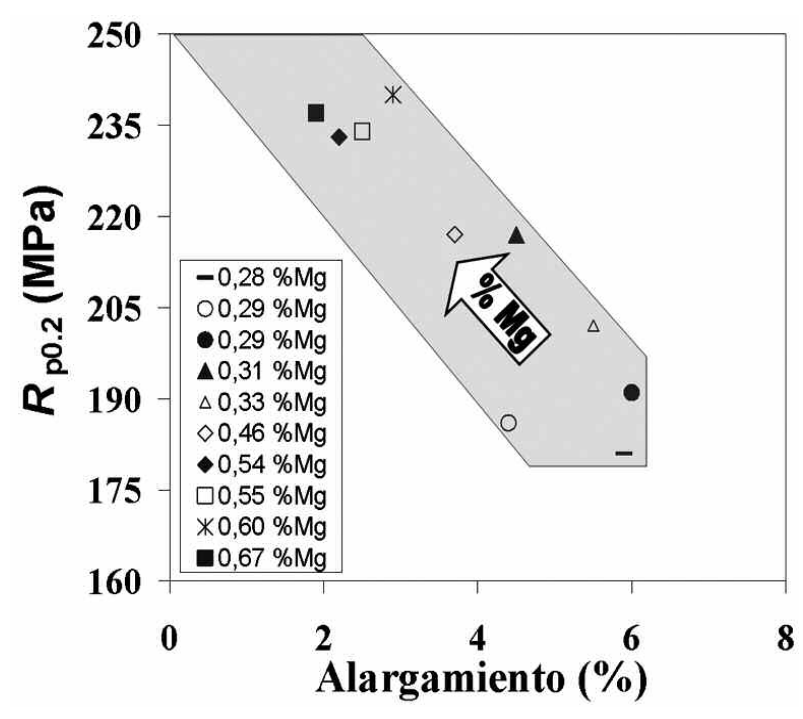

Figura 7. Efecto del porcentaje de magnesio en el límite elástico y el alargamiento para la serie II tratamiento T6.

Figure 7. Effect of magnesium content in yield strength and elongation for the series II after T6 treatment.

\section{CONCLUSIONES}

Se ha estudiado la incidencia del tratamiento térmico (T6) en las propiedades mecánicas para la aleación A356 y, también, la influencia del contenido de magnesio para la aleación AlSi7Mg, cubriendo tanto la aleación A356 como la A357 bajo un mismo tratamiento térmico. Se han utilizado varios índices de calidad que permiten seleccionar un tratamiento 


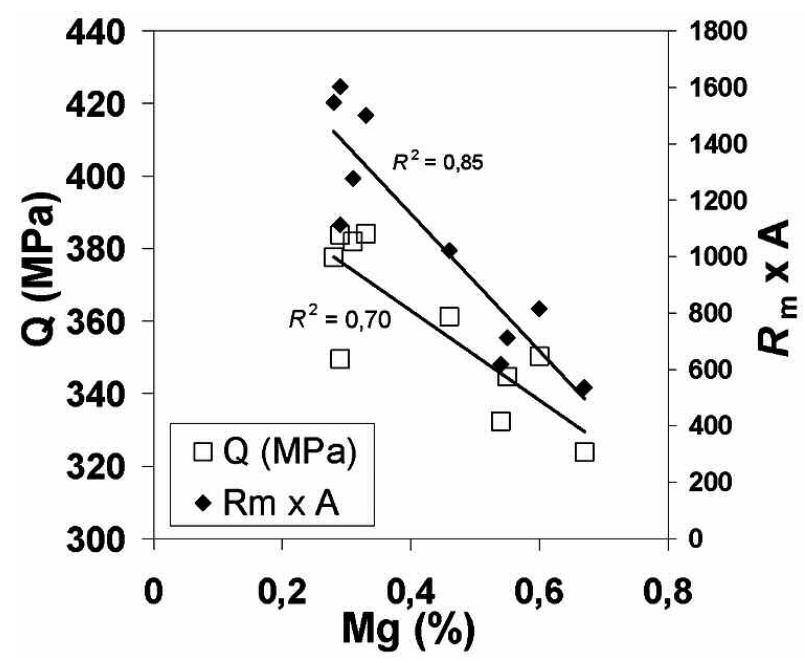

Figura 8. Dependencia $R_{\mathrm{m}} \times A$ y $Q$ en función del porcentaje de magnesio.

Figure 8. Dependence of $R_{m} \times A$ and $Q$ as a function of magnesium content.

térmico óptimo para cada aplicación. Las conclusiones obtenidas en este estudio son las siguientes:

- Para un mismo tiempo de maduración artificial, cuanto menor es la temperatura de precipitación menores son límite elástico, carga máxima y dureza, pero mayor es el alargamiento.

- En el rango de temperaturas estudiadas (155 $\left.170^{\circ} \mathrm{C}\right)$, conforme el tiempo de precipitación incrementa se observa un aumento de límite elástico, carga máxima y dureza, mientras que el alargamiento decrece.

- El valor del ratio $R_{\mathrm{p} 0,2} / R_{\mathrm{m}}$ aumenta conforme aumenta la temperatura y el tiempo de precipitación.

- Los valores máximos del índice Q responden a la temperatura de precipitación de $160{ }^{\circ} \mathrm{C}$, mientras que los mejores valores $R_{\mathrm{m}} \times \mathrm{A}$ responden a la temperatura de $155^{\circ} \mathrm{C}$. El tratamiento precipitación a $155^{\circ} \mathrm{C}$ durante $5,5 \mathrm{~h}$ presenta una buena combinación de propiedades e índices de calidad $Q$ y $R_{\mathrm{m}} \times A$.

- Se observa un aumento de límite elástico y de carga de rotura, a costa de alargamiento, con el aumento del contenido de magnesio.

- Se han obtenido ecuaciones que permiten estimar, en función de los requerimientos de la pieza, el contenido de magnesio óptimo.

\section{Agradecimientos}

Los autores agradecen al Gobierno Central (Proyecto: MITYC 2008: DEX 600800-2008-66) y al Gobierno
Vasco (Proyecto: Manufacturing 0.0, Etortek 2008) la financiación de los proyectos de investigación que ha dado lugar a este trabajo. Los autores, también, agradecen al Prof. Ignacio Alfaro su inestimable ayuda en el análisis de los resultados.

\section{REFERENCIAS}

[1] J.E. Gruzleski, y B.M Closset, The Treatment of Liquid Aluminum - Silicon Alloys, $1^{\underline{a}}$ edición, American Foundrymen's Society Inc., Des Plaines, Illinois, EE. UU., 1990, pp. 25-141.

[2] B. Günther y H. Jürgens, Giesserei 71 (1984) 928-931.

[3] R.I. MacKay, J.H. Djurdjevic, J.H. Sokolowski y W.J. Evans, AFS Trans. 24 (2000) 511-520.

[4] H. Jiang, J.H. Sokolowski, M.B. Djurdjevic y W.J. Evans, AFS Trans. 23 (2000) 505-510.

[5] L. Heusler, Giesserei 82 (1997) 66-73.

[6] A. Apelian, G.K. Sigworth, y K.R. Whaler, AFS Trans. 161 (1984) 297-307.

[7] W. Menk, M. Speidel y R. Döpp, Giesserei 79 (1992) 125-134.

[8] D.G. Ibarra, Tesis Doctoral, Departament of Mining and Metallurgical Engineering, McGill Universidad de Montreal, Canada, 1999.

[9] S. Argyropoulos, B. Closset, J.E. Gruzleski, y H. Oger, AFS Trans. 91 (1983) 35-358.

[10] J. Gilbert, Aluminum Alloys and Tempers, $1^{\underline{a}}$ edición, Ed. ASM International, Ohio, EE. UU., 2000, pp. 1-258.

[11] J. Gilberte y E.L. Rooy, Aluminum Alloy Castings, Properties, Processes and Applications, $2^{a}$ edición, Ed. ASM International, Ohio, EE. UU., 2005, pp. 1-340.

[12] S. Shivkumar, C. Keller y D. Apelian, AFS Trans. 98 (1990) 905-911.

[13] D. Apelian, S. Shivkumar y G. Sigworth, AFS Trans. 97 (1989) 727-742.

[14] S. Shivkumar, S. Ricci, Jr., B. Steenhoff, D. Apelian y G. Sigworth, AFS Trans. 97 (1989) 791-810.

[15] C.H. Caceres y Q.G. Wang, AFS Trans. 104, (1996) 1.039-1.043.

[16] M. Drouzy, S. Jacob y M. Richard, Fonderie 355 (1976) 139-147.

[17] M. Drouzy, S. Jacob y M. Richard, AFS Int. Cast Met. Res. J. 5 (1980) 43-50.

[18] A.I. Fernández-Calvo, J. Barrenengoa, A. Loizaga y A. Niklas, XI Cong. Nac. de Materiales, Libro de Resúmenes y CD, Zaragoza, España, Ed. Universidad de Zaragoza, Zaragoza, España, 2010, com-185, p. 262.

[19] A. Loizaga, A. Niklas, A.I. Fernández-Calvo, J. Lacaze, Int. J. Cast Metal Res. 22 (2009) 345-352. 